\title{
Determinación de sulfatos en cementos y yeso
}

\author{
N. N. BASARGIN Y T. G. AKIMOVA \\ Cement and Lime Manufacture, n० 5, septiembre 1969, pág. 95 \\ (Trasladado de la revista rusa Tsement, $n^{\circ} 6,1968$ )
}

Este trabajo describe un nuevo indicador que puede emplearse para la determinación volumétrica rápida de sulfato en cementos y yeso.

Hay métodos bien conocidos para determinar sulfatos basados en el cambio de ion (1), (2), (3) y (4), así como la espectroscopía infrarroja (5).

De éstos, el método del cambio de ion con valoración alcalimétrica del sulfato merece particular atención (1).

Se ha desarrollado un nuevo método rápido que es diferente, en principio, de los anteriores. Se basa en una determinación volumétrica de sulfato empleando un nuevo indicador, NITCROMAZO (6) y (7), que permite la determinación rápida y exacta de sulfato en cementos y yeso sin necesidad de aparatos complicados. El método lleva consigo la supresión de calcio y magnesio por iones fluoruro (por adición de $\mathrm{HF}$ o $\mathrm{NH}_{4} \mathrm{~F}$ ) y la valoración directa de sulfatos con solución de cloruro bárico standard a un $\mathrm{pH}$ de 1,7 a 2,0 empleando la sal de bario de nitcromazo como indicador. Los ensayos han demostrado que una gran concentración de ácido bórico no interfiere la valoración. Las propiedades del nitcromazo y su síntesis se describen en publicaciones previas (7) y (8).

\section{Determinación de sulfato en cemento}

Una muestra de cemento $(0,800 \mathrm{~g})$ y agua $(90 \mathrm{ml})$ se coloca en un vaso cerrado y calentado sobre un baño de agua durante 15-20 minutos. La mezcla se enfría y filtra en un matraz aforado de 100 c.c. El residuo se lava dos veces con agua, manteniendo el volumen total por debajo del enrase del matraz. La solución se acidula con unas gotas de $\mathrm{ClH} 6 \mathrm{~N}$ para dar un valor de $\mathrm{pH}$ de 4 a 5 (empleando papel indicador como comprobante) y se enrasa.

Una fracción ( 5 c.c.) se traslada a un vaso de 50 c.c. y se trata con $\mathrm{ClH}$ ( 2 c.c. $0,1 \mathrm{~N}$ ), agua (2 c.c.), solución de $\mathrm{FNH}_{4}$ (1 c.c. $0,1 \mathrm{~N}$ ), solución acuosa de nitcromazo (una gota, 0,2 \%) y alcohol etílico (8 c.c.). Esta solución se valora luego con solución standard de $\mathrm{Cl}_{2} \mathrm{Ba}$ $0,02 \mathrm{~N}$. La valoración se lleva a cabo lentamente durante 15 a 20 segundos, agitando después de añadir cada 2 ó 3 gotas, hasta que el color cambia a un azul persistente. $\mathrm{El} \mathrm{FH}$ (5 c.c.) puede sustituir a las soluciones de $\mathrm{FNH}_{4}$ y ClH. La solución de $\mathrm{FH}$ es $0,02 \mathrm{~N}$.

Un ensayo en blanco se lleva a cabo como sigue: Se introduce $\mathrm{ClH}$ ( 1 c.c. $0,1 \mathrm{~N})$ en un vaso de 50 c.c. y se trata con agua ( 8 c.c.), $\mathrm{FNH}_{4}(1$ c.c. $0,1 \mathrm{~N})$, solución acuosa de nitcromazo (una gota, $0,2 \%$ ) y alcohol etílico o acetona (8 c.c.). A continuación se añaden una o más gotas de la solución de cloruro bárico standard para producir color azul. 
El contenido en sulfatos se calcula por la fórmula:

donde:

$$
\mathrm{SO}_{3}(\text { por ciento })=\frac{(a-c) \mathrm{K}}{\mathrm{W}} \cdot 100 ;
$$

$$
\begin{aligned}
a= & \text { cantidad de solución de } \mathrm{Cl}_{2} \mathrm{Ba} \text { standard utilizada en la valoración; } \\
\mathrm{c}= & \text { cantidad de solución de } \mathrm{Cl}_{2} \mathrm{Ba} \text { standard empleada en la determinación en } \\
& \text { blanco; } \\
\mathrm{W}= & \text { peso de muestra en la parte alícuota }(\mathrm{ml}) ; \\
\mathrm{K}= & \mathrm{K}_{1} \times \mathrm{f}_{1} ; \\
\mathrm{K}_{1}= & \text { diferencia en normalidad de la solución de } \mathrm{Cl}_{2} \mathrm{Ba} \text { de } 0,02 \mathrm{~N} \text { (es decir, fac- } \\
& \text { tor de la solución); } \\
\mathrm{f}_{1}= & \text { cantidad de } \mathrm{SO}_{3} \text { equivalente a } 1 \text { c.c. de solución de } \mathrm{Cl}_{2} \mathrm{Ba} 0,02 \mathrm{~N}(=0,80) .
\end{aligned}
$$

La solución de cloruro bárico se contrasta frente a una solución de ácido sulfúrico standard de la misma concentración, sin adición de soluciones de $\mathrm{FNH}_{4}$ o $\mathrm{FH}$. El $\mathrm{FNH}_{4}$ o el $\mathrm{FH}$ se añaden para reaccionar con el calcio que, en forma no combinada, tiende a afectar el color del indicador.

Cuando se valora en presencia de $\mathrm{FH}$, el indicador cambia el color más marcadamente del violeta al azul y la parte alícuota puede aumentarse a 10 c.c., aun empleando 5 c.c. de solución $0,2 \mathrm{~N}$ de $\mathrm{FH}$.

\section{Determinación de sulfato en yeso}

Una muestra de yeso $(0,2 \mathrm{~g})$ y agua ( 150 c.c.) se calienta en un vaso de 250 c.c. en un baño de agua de 15 a 20 minutos. La solución se enfría y filtra y el residuo se lava con 20-30 c.c. de agua. Después se ajusta el volumen a 250 c.c. en un matraz aforado.

Una parte alícuota ( 5 c.c.) se trasvasa a un vaso de 50 c.c. y se trata con $\mathrm{ClH}$ ( 1 c.c. $0,1 \mathrm{~N}), \mathrm{FNH}_{4}(1$ c.c. $0,1 \mathrm{~N})$, agua (3 c.c.), solución acuosa de nitcromazo (una gota, 0,2 \%) y alcohol etílico o acetona (8 c.c.).

La muestra se valora lentamente frente a una solución standard 0,02 $\mathrm{N}$ de cloruro bárico desde una microbureta, hasta que el color cambia de un azul-violeta a un azul persistente.

Se lleva a cabo una determinación en blanco, y el contenido de $\mathrm{SO}_{3}$ se calcula a partir de la fórmula dada anteriormente para cemento.

Para una valoración se necesitan 5 a 7 minutos, y la determinación total dura alrededor de 30 minutos.

\section{B I B L I O G R A F I A}

(1) I. V. Bogdanova. Zhur. anal. khimii, 14, 373 (1959)

(2) L. A. Robins and T. D. Wheelock. Anal. Chem, 36, 429 (1964)

(3) Chan Kal-Nan. Tianzhu cerilo gongue, 31, 35 (1965)

(4) Hu Yuntsu. Jour. Chinese Sil. Soc., 4, 152 (1965)

(5) H. E. Sohweite, and E. Niel. Zement-Kalk-Gips., 18, 157 (1965)

(6) V. I. KuzNetsov and N. N. BASARGiN. Zavod. lab, 21, 473 (1966)

(7) N. N. BASARgin and K. F. Novikova. Zhur. anal. khimii, 21, 473 (1966)

(8) B. I. KuzNetsov and N. N. Basargin. Bulletin: Methods of making chemical

reagents and preparations. Moscow, pub. IREA, issue 13, page 87 (1965). 